\title{
Battery Capacity Estimation of Low-Earth Orbit Satellite Application
}

\author{
Myungsoo Jun ${ }^{1}$, Kandler Smith ${ }^{2}$, Eric Wood ${ }^{3}$, and Marshall .C. Smart ${ }^{4}$ \\ 1,2,3 National Renewable Energy Laboratory, Golden, CO, 80401, USA \\ myungsoo.jun@nrel.gov, kandler.smith@nrel.gov,eric.wood@nrel.gov \\ ${ }^{4}$ Jet Propulsion Laboratory, California Institute of Technology, Pasadena, CA 91109, USA \\ marshall.c.smart@jpl.nasa.gov
}

\begin{abstract}
Simultaneous estimation of the battery capacity and stateof-charge is a difficult problem because they are dependent on each other and neither is directly measurable. This paper proposes a particle filtering approach for the estimation of the battery state-of-charge and a statistical method to estimate the battery capacity. Two different methods and time scales have been used for this estimation in order to reduce the dependency on each other. The algorithms are validated using experimental data from $\mathrm{A} 123$ graphite/ $/ \mathrm{LiFePO}$ lithium ion commercial-off-the-shelf cells, aged under partial depth-ofdischarge cycling as encountered in low-earth-orbit satellite applications. The model-based method is extensible to battery applications with arbitrary duty-cycles.
\end{abstract}

\section{INTRODUCTION}

Health and lifetime uncertainty presents a major barrier to the deployment of lithium-ion (Li-ion) batteries in large-scale aerospace, electric vehicle, and electrical grid applications with stringent life requirements. In the satellite industry, for example, the high cost of launch and the inability to make repairs once in orbit dictate the use of mature battery technologies with conservative duty-cycles to reduce risk. If battery health could be precisely tracked on orbit, the duty-cycle might be tailored to best utilize the remaining life and maximize the value of the investment. Similar opportunities may exist for electric vehicles to maximize battery lifetime by intelligently selecting driving routes and charging strategies. Markets for used electric vehicles and batteries also require accurate battery health assessment to mature to their full potential.

The field of prognostics and health management offers gen-

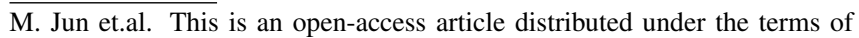
the Creative Commons Attribution 3.0 United States License, which permits unrestricted use, distribution, and reproduction in any medium, provided the original author and source are credited.
}

eral approaches for combining real-time measurements, models and estimation algorithms to track the health and predict the remaining lifetime of batteries (Sheppard, Wilmering, \& Kaufman, 2009; Goebel, 2010). Relevant performance/health metrics for battery applications are available power and energy. These can be expressed in terms of battery internal resistance and amp-hour (Ah) capacity, respectively. Battery models are needed to relate capacity and resistance to the current, voltage, and temperature measurement signals available in real-time. For regular predictable duty-cycles such as in unmanned aerial vehicles (Goebel, Saha, Saxena, Celaya, \& Christophersen, 2008), simple algebraic relationships between current and voltage may be sufficient. For uncertain duty-cycles such as for electric vehicles, a dynamic model of the current and voltage relationship is necessary. Dynamic models can be in the form of circuit analogs (Verbrugge \& Koch, 2006; Plett, 2006), or reduced order physics-based models (Santhanagopalan, Zhang, Kumaresan, \& White, 2008; Smith, Rahn, \& Wang, 2007; Smith, 2010; J. L. Lee, Chemistruck, \& Plett, 2012). Physics-based approaches remain their own active subject of research and thus the simpler circuit model is applied in this work.

State-of-charge (SOC) is usually formulated as a reference model state and can be estimated by using various state estimation methods such as extended Kalman filter (Plett, 2004; J. Lee, Nam, \& Cho, 2007; Charkhgard \& Farrokhi, 2010; Kim \& Cho, 2011; Hu, Youn, \& Chung, 2012), unscented Kalman filter (Plett, 2006; Sun, Hu, Zou, \& Li, 2011) or cubature Kalman filter (Chen, 2012). Those SOC estimation methods work well in certain situations but would not perform properly in other situations. Extended Kalman filters are prone to linearization errors and both extended Kalman filters and unscented Kalman filters are limited to systems with Gaussian noise distribution. Similar to Kalman filters, particle filters belong to the class of Bayesian estimation methods, but can deal with nonlinear systems with non-Gaussian noise without linearization (Sanjeev Arulampalam, Maskell, Gor- 


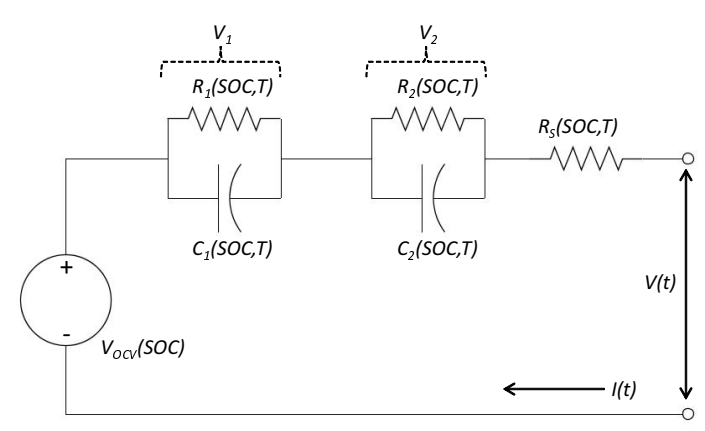

Figure 1. Second order circuit model of a battery

don, \& Clapp, 2002). They have been successfully applied to many problems with nonlinear dynamics such as computer vision (Isard \& Blake, 1998), speech recognition (Vermaak, Andrieu, Doucet, \& Godsill, 2002), robotics (Schulz, Burgard, Fox, \& Cremers, 2001), etc. Furthermore, very little work has been done in SOC estimation in conjunction with simultaneous estimation of time-varying battery capacity. This paper proposes a method to estimate both SOC and battery capacity by using a particle filtering approach.

Unlike in the laboratory, in an application environment it is infeasible to completely discharge the battery to obtain a full "ground-truth" measurement of battery total capacity. A key question explored in this paper is to what extent battery total amp-hour (Ah) capacity can be estimated based on only partial discharge data. In addition, estimation of battery capacity using partial discharge data is particularly challenging for Li-ion chemistries with a flat open-circuit voltage relationship versus SOC (Plett, 2011). Such is the case for the Li-ion graphite/iron-phosphate chemistry investigated in the present work.

\section{Circuit Model}

For the reference model, a second-order circuit model is used in this work as shown in Figure 1. While the battery is an infinite-dimensional system, the two time constants of the second order circuit model provide reasonable approximation of voltage/current dynamics for the present application. The state-space equation of this circuit model is expressed as follows:

$$
\begin{aligned}
& {\left[\begin{array}{c}
S \dot{O} C(t) \\
\dot{V}_{1}(t) \\
\dot{V}_{2}(t)
\end{array}\right]=\left[\begin{array}{ccc}
0 & 0 & 0 \\
0 & -\frac{1}{R_{1} C_{1}} & 0 \\
0 & 0 & -\frac{1}{R_{2} C_{2}}
\end{array}\right]\left[\begin{array}{c}
S O C(t) \\
V_{1}(t) \\
V_{2}(t)
\end{array}\right]} \\
& +\left[\begin{array}{lll}
-\frac{1}{Q} & -\frac{1}{C_{1}} & -\frac{1}{C_{2}}
\end{array}\right]^{T} I(t)+n_{1}(t) \\
& V_{\text {out }}(t)=V_{\text {ocv }}(S O C)-V_{1}(t)-V_{2}(t)- \\
& R_{s} \cdot I(t)+v(t) \text {. }
\end{aligned}
$$

where $Q$ denotes the battery capacity. The values of the parameters $R_{1}, R_{2}, R_{s}, C_{1}$ and $C_{2}$ depend on SOC and time and $Q$ depends on time. (Since the satellite battery considered in this work operates under nearly isothermal conditions, temperature dependency is neglected.)

Measurements of resistance versus SOC exhibit a bathtub shape, with small resistance at mid-SOCs increasing to larger values at low and high SOC extremes. This parametric dependence of $R_{1}, R_{2}$ and $R_{s}$ on SOC is captured in Eq. (3)-(5)

$$
\begin{aligned}
& R_{1}(S O C(t))=a_{r 1}\left(1+b_{r 1} \cdot\left|S O C-c_{r 1}\right|^{d_{r 1}}\right) \\
& R_{2}(S O C(t))=a_{r 2}\left(1+b_{r 2} \cdot\left|S O C-c_{r 2}\right|^{d_{r 2}}\right) \\
& R_{s}(S O C(t))=a_{r s}\left(1+b_{r s} \cdot\left|S O C-c_{r s}\right|^{d_{r s}}\right) .
\end{aligned}
$$

As the battery ages, the values of $Q$ slowly decrease and the resistance values slowly increase over time. Since the battery may not be exercised over its entire SOC range in an actual application, only the three relative resistance parameters $a_{r 1}$, $a_{r 2}$ and $a_{r s}$ are estimated along with the battery capacity. The dynamics of these time-varying parameters can be formulated by:

$$
\begin{aligned}
& {\left[\begin{array}{c}
Q(k+1) \\
a_{r 1}(k+1) \\
a_{r 2}(k+1) \\
a_{r s}(k+1)
\end{array}\right]=M\left[\begin{array}{c}
Q(k) \\
a_{r 1}(k) \\
a_{r 2}(k) \\
a_{r s}(k)
\end{array}\right]+n_{2},} \\
& M=\left[\begin{array}{cccc}
1-\varepsilon_{1} & 0 & 0 & 0 \\
0 & 1+\varepsilon_{2} & 0 & 0 \\
0 & 0 & 1+\varepsilon_{2} & 0 \\
0 & 0 & 0 & 1+\varepsilon_{2}
\end{array}\right]
\end{aligned}
$$

where $\varepsilon_{1}$ and $\varepsilon_{2}$ are small positive constants. We assume $n_{2}$ to be constant. We can reformulate a state-space equation by combining Eq.(1) and Eq. (6). Let $x(k)=$ $\left[\begin{array}{lllllll}S O C(k) & V_{1}(k) & V_{2}(k) & Q(k) & a_{r 1}(k) & a_{r 2}(k) & a_{r s}(k)\end{array}\right]^{T}$ be the augmented state and $\Delta t$ be the sampling time. Then the discrete-time augmented state-space equation of the secondorder circuit model of a battery is expressed as:

$$
\begin{aligned}
x(k+1) & =A x(k)+B I(k)+n(k) \\
V_{\text {out }}(k) & =V_{\text {ocv }}(S O C)-V_{1}(k)-V_{2}(k)- \\
& R_{s} \cdot I(k)+v(k)
\end{aligned}
$$

where

$$
\begin{gathered}
A=\operatorname{diag}\left(1, e^{\lambda_{1} \Delta t}, e^{\lambda_{2} \Delta t}, M\right), \\
B=\left[\begin{array}{c}
-\Delta t / Q \\
R_{1}\left(e^{\lambda_{1} \Delta t}-1\right) \\
R_{2}\left(e^{\lambda_{2} \Delta t}-1\right) \\
0 \\
0 \\
0 \\
0
\end{array}\right], \\
n(k)=\operatorname{diag}\left(n_{1}(k), n_{2}\right) .
\end{gathered}
$$




\section{Particle Filter}

Particle filtering is a method used to approximate the probability density $f_{k}$ of the state $x_{k}$ conditioned on the observations $y_{0}, \cdots, y_{k}{ }^{1}$. Consider the following nonlinear system:

$$
\begin{aligned}
& x_{k}=g\left(x_{k-1}, u_{k-1}\right)+n_{k} \\
& y_{k}=h\left(x_{k}, u_{k}\right)+v_{k} .
\end{aligned}
$$

where $x_{k}$ is the state, $y_{k}$ is the measurement, $n_{k}$ is the process noise, and $v_{k}$ is the measurement noise. Suppose that $f_{k-1}=p\left(x_{k-1} \mid y_{0}, \cdots, y_{k-1}\right)$ is known. Then the a priori distribution of the state $x_{k}$ can be derived via the ChapmanKologorov equation ${ }^{2}$

$$
p\left(x_{k} \mid y_{0}, \cdots, y_{k-1}\right)=\int p\left(x_{k} \mid x_{k-1}\right) f_{k-1} d x_{k-1}
$$

where $p\left(x_{k} \mid x_{k-1}\right)$ represents state transition over time and is determined by the process model (9) and the distribution of the process noise $n_{k}$. Note that $f_{k-1}$ is a function of $x_{k-1}$. This step is called prediction or time propagation. When the observation $y_{k}$ at time $k$ is made, the a priori distribution is updated using Bayes' rule:

$$
\begin{aligned}
f_{k} & =p\left(x_{k} \mid y_{0}, \cdots, y_{k}\right) \\
& =\frac{p\left(y_{k} \mid x_{k}\right) \cdot p\left(x_{k} \mid y_{0}, \cdots, y_{k-1}\right)}{\int p\left(y_{k} \mid x_{k}\right) \cdot p\left(x_{k} \mid y_{0}, \cdots, y_{k-1}\right) d x_{k}} \\
& =\frac{p\left(y_{k} \mid x_{k}\right) \cdot p\left(x_{k} \mid y_{0}, \cdots, y_{k-1}\right)}{p\left(y_{k} \mid y_{0}, \cdots, y_{k-1}\right)} .
\end{aligned}
$$

This step is called the measurement update as the measurement data $y_{0}, \cdots, y_{k}$ are used to obtain the a posteriori distribution $f_{k}=p\left(x_{k} \mid y_{0}, \cdots, y_{k}\right)$. The distribution $p\left(y_{k} \mid x_{k}\right)$ can be obtained from the measurement equations (10) and the distribution of the measurement noise $v_{k}$.

Particle filters approximate $f_{k}$ by a set of weighted samples or particles $x_{k}^{i}, i=1, \cdots, N$, where $N$ is the number of particles. For more details about particle filters and sequential Monte Carlo methods, refer to (Sanjeev Arulampalam et al., 2002).

In this paper, sampling importance resampling is used for resampling of the particle filter to reduce degeneracy. The algorithm for the particle filter used in the simulations is given in the following:

1. Initialization: $k=0$

- Draw $x_{0}^{i}, i=1, \cdots, N$ from the initial prior $f_{0}=$ $p\left(x_{0}\right)$.

2. For $k=1,2, \cdots$

(a) Importance Sampling Step

\footnotetext{
$\overline{{ }^{1} \text { Let the subscript }} k$ denote discrete time $k$ for simple notation, i.e., $x_{k}=$ $x(k)$

${ }^{2} p(x \mid y)$ means $p(X=x \mid Y=y)$ for simplicity of notation where $X$ and

$Y$ are random variables and $x$ and $y$ are their realizations.
}

- State transition: For $i=1, \cdots, N$, draw $x_{k}^{i}$ from $p\left(x_{k}^{i} \mid x_{k-1}^{i}\right)$, viz., from Eq. (9).

- Measurement update and likelihood calculation: For $i=1, \cdots, N$, evaluate likelihood by calculating $w_{k}^{i}=p\left(y_{k} \mid x_{k}^{i}\right)$ after the measurement $y_{k}$ is available.

- Normalization: Normalize the importance weights $\tilde{w}_{k}^{i}=w_{k}^{i} / \sum_{j} w_{k}^{i}$.

\section{(b) Resampling}

- Resample $\hat{x}_{k}^{i}$ using updated weights $\tilde{w}_{k}^{i}$.

- $\quad$ Set a new weight $w_{k}^{i}=1 / N$ for $i=1, \cdots, N$.

\section{Capacity Estimation}

The simultaneous estimation of the battery capacity and SOC is difficult because they are dependent on each other by the relation

$$
S O C(t)=S O C(0)-\int_{0}^{t} \frac{I(\tau)}{Q} d \tau
$$

Therefore, if the changes in the battery capacity $Q$ are not reflected properly, the calculation of SOC based on Eq. (13) is subject to errors even though the measurement of $I(t)$ is accurate. This paper proposes a novel method to estimate the battery capacity and SOC simultaneously using a particle filter and statistical approach.

The actual value of $Q$ in real situations changes very slowly over time. This paper utilizes past statistical information for an estimate of $Q$ at a longer interval than the sampling time. Let $m \gg 1$ be an integer and $T=m \Delta t$. The battery capacity is estimated at every $T$ and the value of $Q$ in Eq. (1) is set to the estimated battery capacity $\hat{Q}$ at every $T$ other than $\Delta t$.

The estimate of $x_{k}$ by the particle filter is the weighted sample mean of the particles, that is, $\hat{x}_{k}=\sum_{i=1}^{N} w_{k}^{i} x_{k}^{i}$ and the $i$ - $j$-th element $q_{k}(i, j)$ of the weighted covariance matrix $\mathbf{Q}_{k}$ are

$$
\begin{aligned}
q_{k}(i, j)= & \frac{\sum_{n=1}^{N} w_{k}^{n}}{\left(\sum_{n=1}^{N} w_{k}^{n}\right)^{2}-\sum_{n=1}^{N}\left(w_{k}^{n}\right)^{2}} \times \\
& \sum_{n=1}^{N} w_{k}^{n}\left(x_{k}^{n}(i)-\hat{x}_{k}(i)\right)\left(x_{k}^{n}(j)-\hat{x}_{k}(j)\right)
\end{aligned}
$$

where $x_{k}^{n}(i)$ and $\hat{x}_{k}(i)$ are the $i$-th elements of the vectors $x_{k}^{n}$ and $\hat{x}_{k}(i)$, respectively. The value of $q_{k}(4,4)$ implies an estimation error for $x_{k}(4)=Q(k)$ and the degree of confidence can be represented by the reciprocal of $q_{k}(4,4)$. Thus, the paper uses as estimate of $Q$

$$
\begin{array}{r}
\hat{Q}(\ell T)=\sum_{k=(\ell-1) T}^{\ell T} \frac{W_{k}}{\sum_{j=(\ell-1) T}^{\ell T} W_{j}} \sum_{i=1}^{N} w_{k}^{i} x_{k}^{i}(4), \\
\ell=1,2, \cdots
\end{array}
$$




\begin{tabular}{c|c|c|c||c|c|c|c||c|c|c|c}
\hline$a_{r s}$ & $b_{r s}$ & $c_{r s}$ & $d_{r s}$ & $a_{r 1}$ & $b_{r 1}$ & $c_{r 1}$ & $d_{r 1}$ & $a_{r 2}$ & $b_{r 2}$ & $c_{r 2}$ & $d_{r 2}$ \\
\hline 0.0105 & 112.8616 & 0.5221 & 5.5892 & 0.0440 & 0.0176 & 0.6307 & 3.5007 & 0.0157 & 0.0234 & 0.0029 & 7.3141 \\
\hline
\end{tabular}

Table 1. Beginning of life parameter values in Eq.(3)-(5) for $R_{1}, R_{2}$ and $R_{s}$

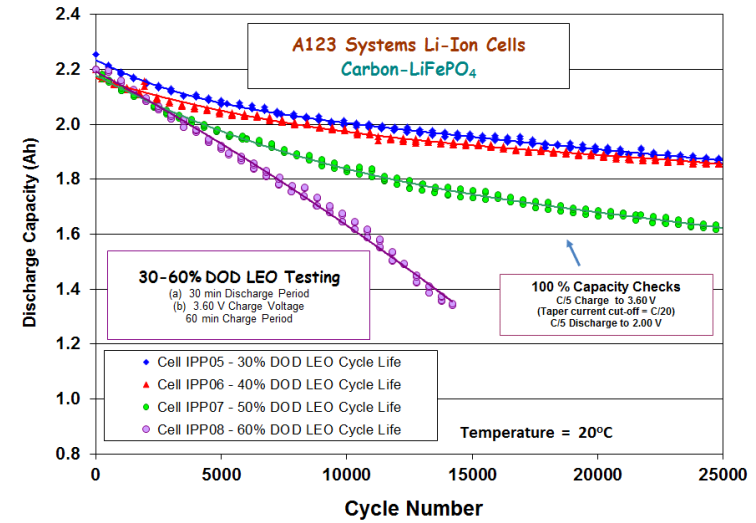

Figure 2. Capacity loss during partial DOD cycling of A123 $\mathrm{LiFePO}_{4}$-based cells (Courtesy Jet Propulsion Laboratory)

where $W_{k}=1 / q_{k}(4,4)$ and the value of $Q(\ell T)$ is reset to a new value of $Q$ in Eq. (1) for every $\ell T, \ell=1,2, \cdots$. This formulation can be interpreted that $W_{k}$ is a weight and Eq (15) a weighted time average and re-initialization of state variables.

\section{RESULTS}

\subsection{Low Earth Orbit Satellite Application}

For the simulations, we used battery data generated at the Jet Propulsion Laboratory. They performed experiments to evaluate the cycle life performance of A123's $26650 \mathrm{LiFePO}_{4}{ }^{-}$ based commercial off-the-shelf cells for potential low earth orbit satellite applications. This testing consists of implementing partial depth-of-discharge (DOD) cycling, with $30 \%, 40 \%, 50 \%$, and $60 \%$ DOD selected. The testing was performed at the room temperature $\left(23^{\circ} \mathrm{C}\right)$ and consisted of a 30-minute discharge period and a 60-minute charge period. The charge and discharge rates were scaled proportionately to the corresponding DOD (i.e., the $30 \%$ DOD test involved using a $0.4 \mathrm{C}$ charge rate and a $0.60 \mathrm{C}$ discharge rate). For operational capacity checks (OPCAPS), full charge and discharge of the battery were conducted every 250 cycles. The plots of battery capacity with respect to cycle number are shown in Figure 2. The degradation of battery capacity is clearly observed from the plot.

The analysis contained in this paper focuses upon the $50 \%$ DOD data from cycle 2723 to 2815 . The battery capacity is reduced to about 2.05 $\mathrm{Ah}$ from the initial 2.2 Ah in the range of these cycles.

Least-square regression was used to provide an initial set

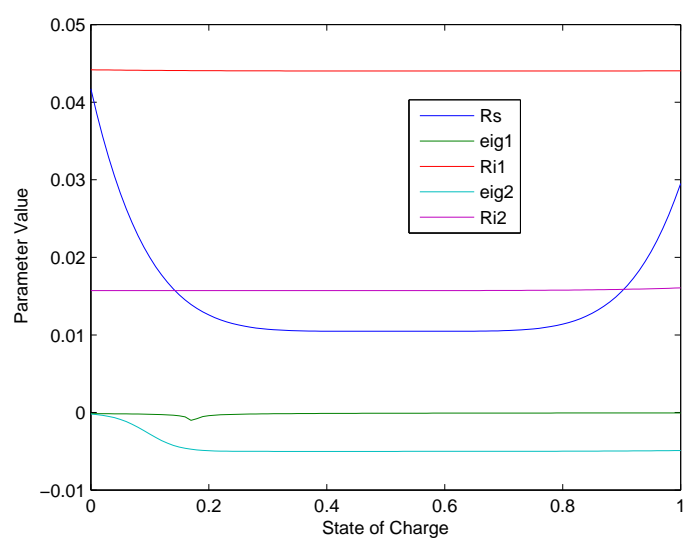

Figure 3. The values of resistances and eigenvalues identified at beginning of life by minimizing the square of voltage error between model and HPPC test data

of parameters representing the battery at beginning of life. Specifically, the Nelder-Mead algorithm (MATLAB function fminsearch) minimized the square of the error between the model's output voltage and measured voltage from a hybrid pulse power characterization (HPPC) test run on a cell at beginning of life (Danzer \& Hofer, 2008). The beginningof-life values parameterizing $R_{1}, R_{2}$ and $R_{s}$ as functions of SOC are shown in Table 1 and the plots of each resistance and eigenvalue are illustrated in Figure 3. Several nonlinearities arise in the model. Values of open-circuit voltage, $V_{o c v}(S O C)$ in Eq. (2), were taken at $10 \%$ increments in SOC following each one-hour rest period of the HPPC test and were implemented in the model as a look-up table. The nonlinearity in Eq. (1) lies in time-varying parameters, $R_{1}, R_{2}$, and $R_{s}$, which are also dependent on SOC.

The values of the parameters in the particle filter were tuned with simulations. We set the vales of $\varepsilon_{1}$ and $\varepsilon_{2}$ to be 0.00001 . The value of the measurement noise $v$ changes adaptively depending on SOC

$$
v(S O C)= \begin{cases}v_{0}\left(1+m_{v}(0.1-S O C)\right), & 0.1>S O C \\ v_{0}\left(1+m_{v}(S O C-0.9)\right), & 0.9<S O C \\ v_{0}, & \text { otherwise }\end{cases}
$$

where $m_{v}$ is a scaling constant depending on measurement error when the value of SOC is very high or low. The process noise $n_{2}$ is set to be a constant and $n_{1}$ to be a function of SOC

$$
n_{1}(S O C)= \begin{cases}n_{10}, & S O C \geq 0.1 \\ n_{10}\left(1+m_{w}(0.1-S O C)\right), & S O C<0.1\end{cases}
$$




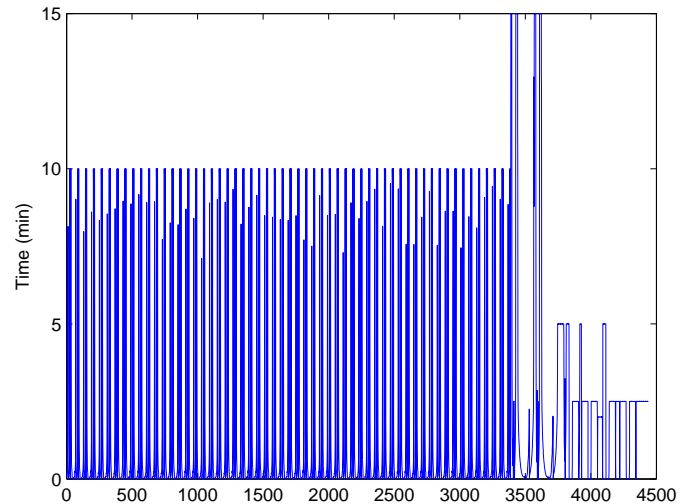

Figure 4. The time intervals between data samples from JPL experiment data

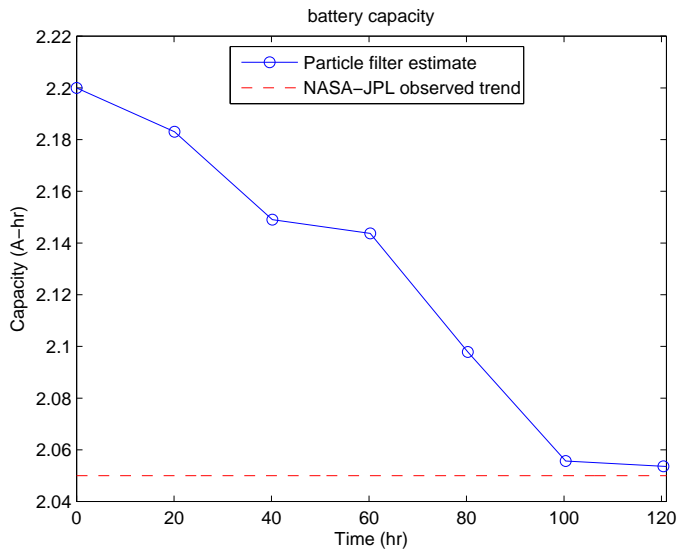

Figure 5. Battery capacity estimation with OPCAPS

where $m_{w}$ is a scaling constant.

The number of particles used in the simulations is 3,000. The sampling time of the filter is dependent on the interval of measurements. The plot of measurement intervals that are used for the simulations in Section 5.1.1 is shown in Figure 4. Measurements were mostly sampled at every 10 minute and the biggest sampling interval is 15 minute. The particle filter used in the simulations performs stratified resampling (Kitagawa, 1996) if

$$
\widehat{N_{e f f}}=\frac{1}{\sum_{i=1}^{N}\left(w_{k}^{i}\right)^{2}}<0.5 N
$$

where $N$ is the number of particles. Otherwise, the particle filter resamples using the normalized importance weight described in Section 3.

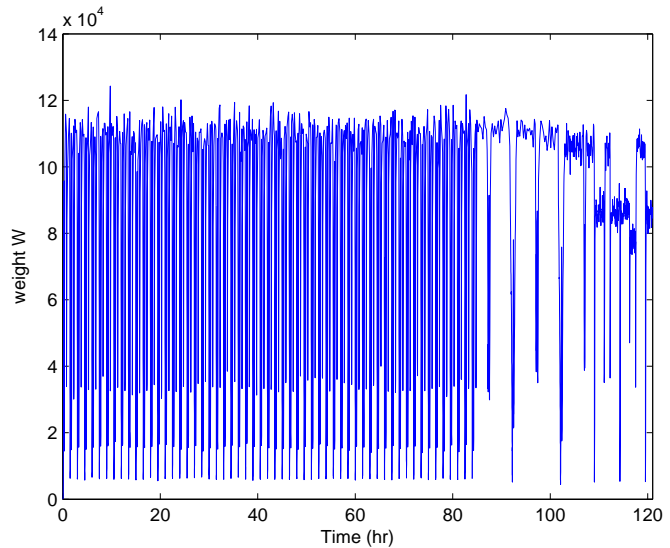

(a) Weight $W_{k}$

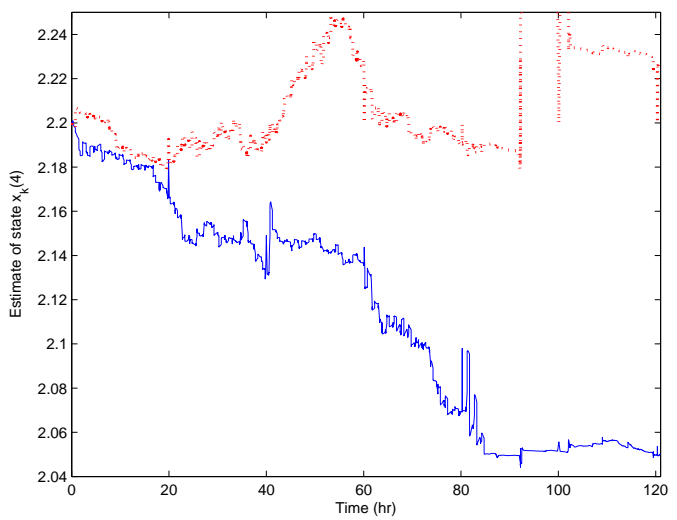

(b) Estimate of $x_{k}(4)$

Figure 6. Weight $W_{k}$ and estimate of $x_{k}(4)=$ $\sum_{i=0}^{N} w_{k}^{i} x_{k}^{i}(4)$

\subsubsection{Simulation with Operational Capacity Checks (OP- CAPS)}

First, we performed simulations with the data from cycle 2773 to 2815 that include OPCAPS. The estimate of the battery capacity was done every 20 hours, that is $T=20 \mathrm{hr}$ in Eq. (15). Figure 5 shows the plot of the battery capacity estimate. The initial value of $Q$ is set to 2.2 at time 0 , which is the initial battery capacity before battery degradation. This initial value is kept until $20 \mathrm{hr}$. At $T=20 \mathrm{hr}$, the battery capacity is estimated to be about 2.1831 and the state variable $Q$ in the particle filter is re-initialized to this value, and so on.

The plots of weight $W_{k}=1 / q_{k}(4,4)$ and the estimate of $x_{k}(4)=\sum_{i=0}^{N} w_{k}^{i} x_{k}^{i}(4)$ that are used for the battery capacity estimation using Eq. (15) are shown in Figure 6a. Figure $6 \mathrm{~b}$ presents estimation of state $x_{k}(4)$ with (solid line) and without (dotted line) the proposed two-time scale method, respectively. It demonstrates that the proposed two-time 


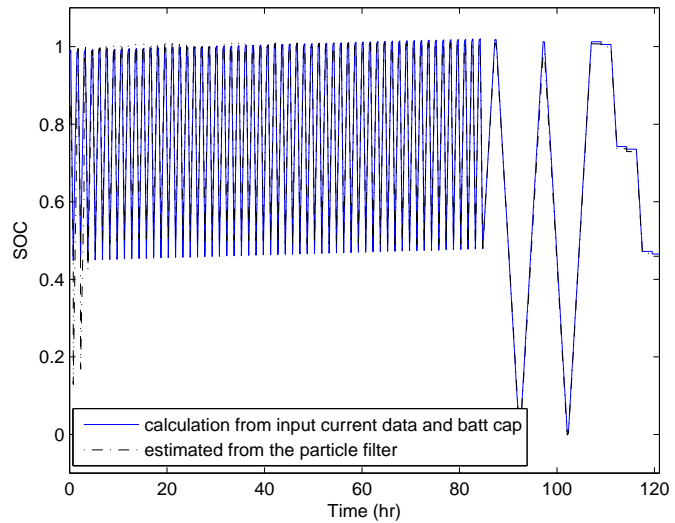

(a) SOC estimation

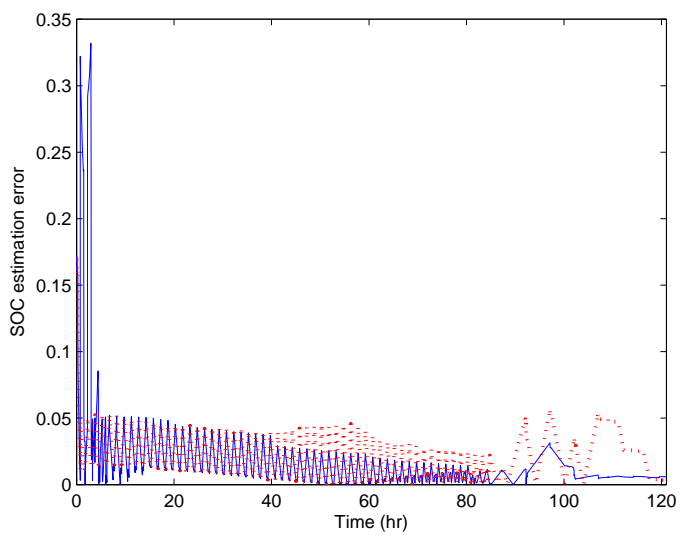

(b) Estimation error

Figure 7. Battery SOC estimation and error with OPCAPS

scale method performs better than particle filtering using augmented state which is usually used for the simultaneous estimation of state and parameters.

The SOC estimate and estimation error are illustrated in Figure 7. The blue solid line in Figure 7a shows the SOC value calculated from input current data and the true battery capacity (2.05 Ah) by using Eq. (13) and the black dash-dotted line illustrates estimated SOC from the particle filter. It can be observed in this plot that the peak value of SOC calculated from Eq. (13) increases over time. This is because measurement errors are accumulated through integration in Eq. (13) and the estimation using the particle filter is more robust to the measurement errors. The estimation error in Figure $7 \mathrm{~b}$ is the difference between the estimate by the particle filter and the calculated value from input current data and the true battery capacity (2.05 Ah) using Eq. (13). The solid line in Figure $7 \mathrm{~b}$ indicates SOC estimation error by using the proposed two-time scale method and the dotted line represents error by particle filtering without two-time scale. It shows

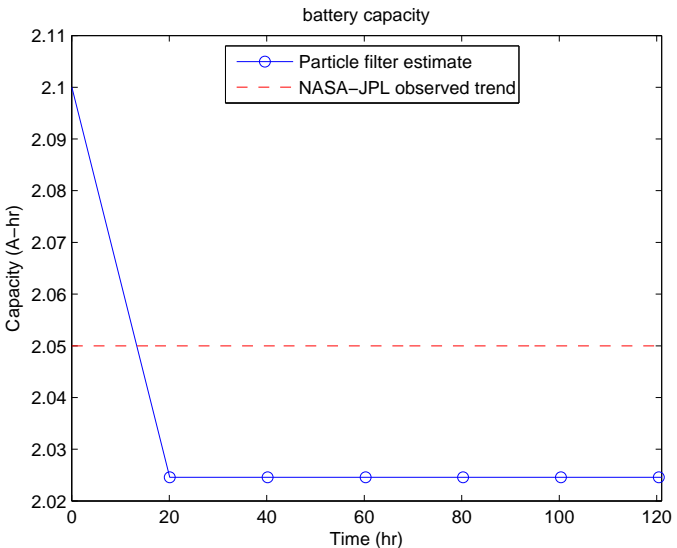

Figure 8 . Battery capacity estimation without OPCAPS

that the error goes below 0.01 (1\%) after about $100 \mathrm{hr}$ by using the proposed method while the error does not decrease without two-time scale method.

\subsubsection{Simulation without Operational Capacity Checks (OPCAPS)}

The second simulation was performed with the data from cycle 2723 to 2806, which does not include OPCAPS and only has repeated charge and discharge with 50\% DOD. The simulation results are shown in Figure 8,10 and 9. In this case, the accumulated error in the SOC calculation by Eq. (13) is more noticeable. However, the SOC estimation using a particle filter oscillates between 0.5 and 1 , which is the expected SOC range with 50\% DOD.

The estimate of the battery capacity converges to about 2.025 $\mathrm{Ah}$, which is a little less than $2.05 \mathrm{Ah}$, the actual capacity. The errors in SOC and the battery capacity estimation without OPCAPS are greater than those with OPCAPS and it took longer time to converge for SOC estimation. However, the error is about $1.2 \%$ and the estimate can be concluded to be accurate even without OPCAPS.

\section{Conclusion}

A method to simultaneously estimate both the capacity and $\mathrm{SOC}$ of a Li-ion battery has been proposed using a particle filtering method for SOC estimation and a statistical approach for the battery capacity. The battery capacity estimation has been performed in a different time scale from the SOC estimation and used accumulated past data from both measurement and the particle filter outputs. The estimated value of the battery capacity has been used to modify the parameter of the battery state-space model. Simulation results showed the robust performance of the algorithm in simultaneous estimation with or without operational capacity checks. The proposed method has been shown to perform better than the particle 


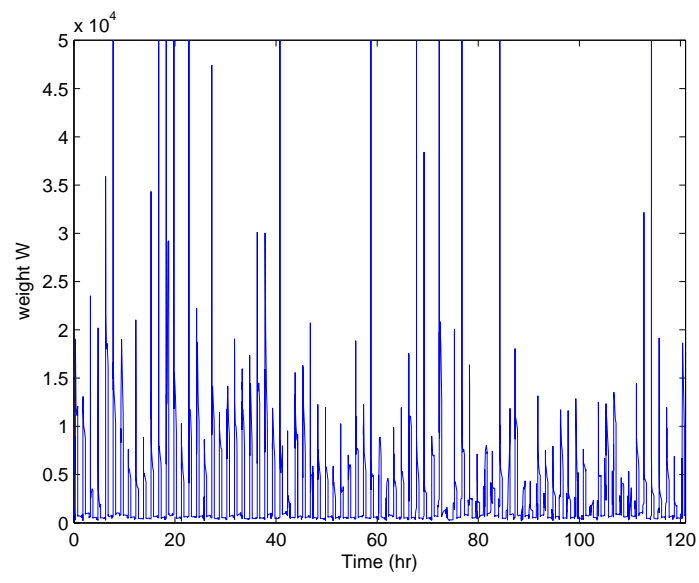

(a)

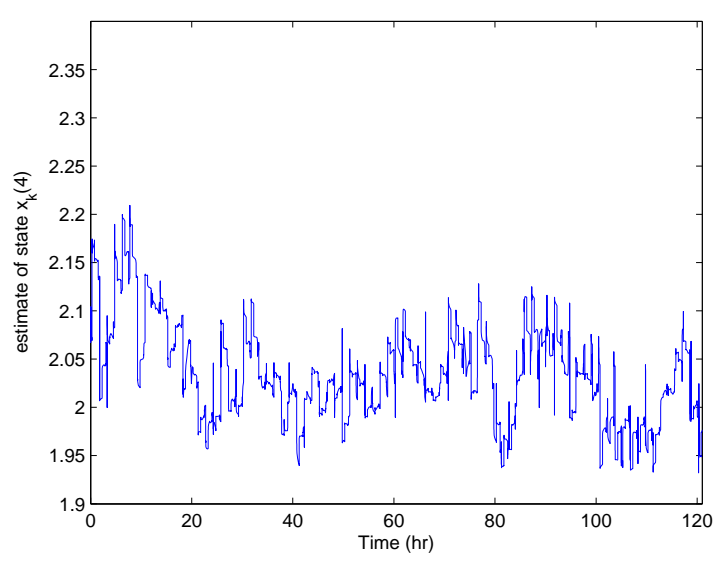

(b)

Figure 9. Weight $W_{k}$ and estimate of $x_{k}(4)=\sum_{i=0}^{N} w_{k}^{i} x_{k}^{i}(4)$

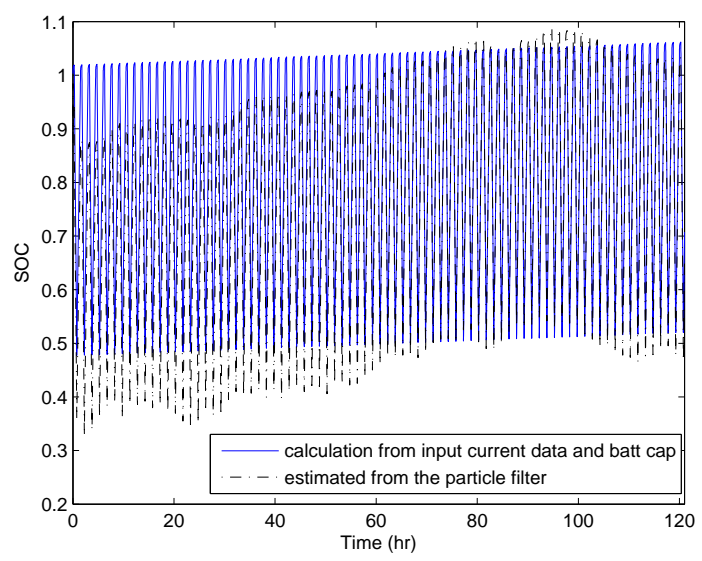

(a) SOC estimation

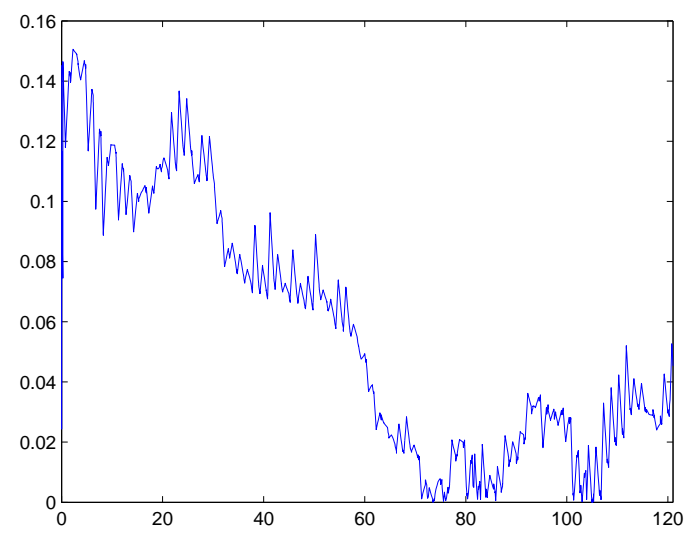

(b) Estimation error

Figure 10. Battery SOC estimation and error without OPCAPS 
filter with one time scale and augmented state. Furthermore, accumulation of bias error over time has been shown to be corrected in SOC estimation with the proposed method.

Due to the high cost of launch, satellite batteries are expected to operate until the end of the satellite's life. Unlike the OPCAP used in laboratory tests, in space the battery can never be fully discharged and hence the battery's total capacity must be indirectly estimated. Trending of battery total capacity over lifetime is important for satellite health management to ensure that no regular partial discharge cycle ever exceeds the present capability of the battery, causing loss of the satellite. The proposed method is adequate for the satellite applications since it estimates the battery capacity and SOC robustly even without OPCAPS and measurement errors are not accumulated in SOC estimation unlike Coulomb count method, which indicates that it is suitable for the applications with long operation time.

\section{ACKNOWLEDGMENT}

M. Jun, K. Smith and E. Wood gratefully acknowledge the National Renewable Energy Laboratory Laboratory-Directed Research and Development program for battery health algorithm development. The work performed by M. C. Smart was carried out at the Jet Propulsion Laboratory, California Institute of Technology, under contract with National Aeronautics and Space Administration (NASA).

\section{REFERENCES}

Charkhgard, M., \& Farrokhi, M. (2010). State-of-Charge Estimation for Lithium-Ion Batteries Using Neural Networks and EKF. IEEE Trans. Industrial Electronics, 57(12), 4178-4187.

Chen, H. (2012, july). Adaptive Cubature Kalman Filter for Nonlinear State and Parameter Estimation. In Proc. of 15th International Conference on Information Fusion. Singapore.

Danzer, M. A., \& Hofer, E. P. (2008). Electrochemical parameter identification - An efficient method for fuel cell impedance characterization. J. Power Sources, 183, 55-61.

Goebel, K. (2010, October). System Health Management: Predicting Failure after Fielding a Product. In IEEE Accelerated Stress Testing and Reliability Workshop. Denver, CO.

Goebel, K., Saha, B., Saxena, A., Celaya, J. R., \& Christophersen, J. (2008, August). Prognostics in Battery Health Management. IEEE Instrumentation \& Measurement Magazine, 11(4), 33-40.
Hu, C., Youn, B. D., \& Chung, J. (2012). A multiscale framework with extended Kalman filter for lithium-ion battery SOC and capacity estimation. J. of Applied Energy, 92, 694-704.

Isard, M., \& Blake, A. (1998). CONDENSATION — conditional density propagation for visual tracking. International Journal of Computer Vision, 29(1), 5-28.

Kim, J., \& Cho, B. H. (2011). State-of-Charge Estimation and State-of-Health Prediction of A Li-Ion Degraded Battery Based on An EKF Combined with A Per-Unit System. IEEE Trans. Vehicular Technology, 60(9), 42494260.

Kitagawa, G. (1996). Monte-Carlo filter and smoother for non-Gaussian nonlinear state space model. J. Comput. Graph. Statist., 1, 1-25.

Lee, J., Nam, O., \& Cho, B. H. (2007). Li-ion battery SOC estimation method based on the reduced order extended Kalman filtering. J. of Power Sources, 174, 9-15.

Lee, J. L., Chemistruck, A., \& Plett, G. L. (2012, December). One-dimensional physics-based reduced-order model of lithium-ion dynamics. J. Power Sources, 220, 430448.

Plett, G. L. (2004). Extended Kalman filtering for battery management systems of LiPB-based HEV battery packs. Part 3: State and parameter estimation. J. of Power Sources, 134(2), 277-292.

Plett, G. L. (2006). Sigma-point Kalman filtering for battery management systems of LiPB-based HEV battery packs. Part 2: Simultaneous state and parameter estimation. J. of Power Sources, 161, 1369-1384.

Plett, G. L. (2011). Recursive approximate weighted total least squares estimation of battery cell total capacity. $J$. of Power Sources, 196, 2319-2331.

Sanjeev Arulampalam, M., Maskell, S., Gordon, N., \& Clapp, T. (2002). A tutorial on particle filters for online nonlinear/non-Gaussian Bayesian tracking. IEEE Trans. on Signal Processing, 50(2), 174-188.

Santhanagopalan, S., Zhang, Q., Kumaresan, K., \& White, R. E. (2008). Parameter estimation and life modeling of lithium-ion cells. J. Electrochem. Soc., 155(4), A345A353.

Schulz, D., Burgard, W., Fox, D., \& Cremers, A. (2001). Tracking multiple moving targets with a mobile robot using particle filters and statistical data association. In Proc. of the IEEE International Conference on 
Robotics and Automation (p. 1665-1670). Seoul, Korea.

Sheppard, J. W., Wilmering, T. J., \& Kaufman, M. A. (2009). IEEE Standards for Prognostics and Health Management. IEEE Aerospace and Electronic Systems Magazine, 24(9), 34-41.

Smith, K. (2010, April). Electrochemical Control of LithiumIon Batteries. IEEE Control Systems Magazine.

Smith, K., Rahn, C. D., \& Wang, C. Y. (2007). ControlOriented 1D Electrochemical Model of Lithium Ion Battery. Energy Conversion and Management, 48(9), 2565-2578.
Sun, F., Hu, X., Zou, Y., \& Li, S. (2011). Adaptive unscented Kalman filtering for state of charge estimation of a lithium-ion battery for electric vehicles. J. of Energy, 36, 3531-3540.

Verbrugge, M. W., \& Koch, B. J. (2006). Generalized recursive algorithm for adaptive multiparameter regression. J. Electrochem. Soc., 153(1), A187-A201.

Vermaak, J., Andrieu, C., Doucet, A., \& Godsill, S. (2002, March). Particle methods for Bayesian modeling and enhancement of speech signals. IEEE Trans. on Speech and Audio Processing, 10, 173-185. 